\title{
Is Toxoplasma gondii infection correlated with nonalcoholic fatty liver disease?- a population-based study
}

\author{
Jiaofeng Huang ${ }^{1}$, Haoyang Zhang ${ }^{2}$, Shiying Liu', Mingfang Wang ${ }^{1}$, Bo Wan ${ }^{3}$, Bharat Velani', Yueyong Zhu' \\ and Su $\operatorname{Lin}^{1 *}$ (D)
}

\begin{abstract}
Background: Previous studies have suggested that Toxoplasma gondii (T. gondii) infection might be associated with fatty liver disease. However, the relationship between non-alcoholic fatty liver disease (NAFLD) and T. gondii infection has not been investigated in a large population. We aimed to study the relationship between those two diseases using a population-based dataset from the United States.

Methods: The data were collected from the third National Health and Nutrition Examination Survey (NHANES III) between 1988 and 1994. Statistical analysis was applied to compare the prevalence of NAFLD in anti-T. gondii antibody-positive participants with antibody-negative ones.

Results: A total of 9465 persons with a mean age of $44.33 \pm 16.21$ years, $46.9 \%$ of which were males, were included in the final analysis. Their mean BMI was $27.60 \pm 5.96 \mathrm{~kg} / \mathrm{m}^{2}$. A total of 2520 participants (26.62\%) were positive for the T. gondii antibody. There was an increasing trend of seroprevalence of $T$. gondii with age ( $P$ for trend $<0.001$ ). The incidence of NAFLD in the seropositive group was higher than that in the seronegative group ( $27.10 \%$ vs 23. $40 \%, p<0.001)$. In addition to this, metabolic biomarkers, including serum lipid, fasting blood-glucose, and uric acid were also significantly higher in the seropositive group. However, multivariate analysis revealed that $T$. gondii infection was not an independent risk factor for NAFLD. Age was independently correlated with both the prevalence of T. gondii and NAFLD.
\end{abstract}

Conclusions: Patients with T. gondii infection may have a higher prevalence of NAFLD. Age may have an effect on the increase of NAFLD in the T. gondii seropositive population.

Keywords: Non-alcoholic fatty liver, Toxoplasma gondii, NHANES

\section{Background}

Toxoplasma gondii (T. gondii) infection is a major global public health problem. Approximately $30 \%$ of the world's population show serological evidence of infection [1]. Although most infections are subclinical and benign, some may cause severe consequences, including lymphadenopathy, hepatitis, ophthalmitis, schizophrenia and other important organ dysfunction [2,3]. When the liver is involved, T. gondii infection can present with hepatomegaly,

\footnotetext{
* Correspondence: sumer5129@fjmu.edu.cn

${ }^{1}$ Department of Liver Research Center, the First Affiliated Hospital of Fujian Medical University, Fuzhou, No. 20, Chazhong Road, Taijiang District, Fuzhou 350001, Fujian, China

Full list of author information is available at the end of the article
}

liver inflammation, liver granuloma formation and cirrhosis $[4,5]$. Several studies demonstrated a higher seroprevalence of $T$. gondii antibody in patients with liver cancer, cirrhosis, acute and chronic hepatitis [4-8].

Non-alcoholic fatty liver disease (NAFLD) is a disease with an excessive accumulation of fat in the liver, with different complications including inflammation, fibrosis, cirrhosis and hepatocellular carcinoma. It is the most common chronic liver disease with a global prevalence of $30 \%$ [9]. A recent study demonstrated that mice infected with $T$. gondii had significant inflammation and steatosis in the liver [10]. Epidemiological data from eastern China showed that the prevalence rate of $T$. gondii was higher in people with liver steatosis $(22.75 \%)$ compared

(C) The Author(s). 2018 Open Access This article is distributed under the terms of the Creative Commons Attribution 4.0 International License (http://creativecommons.org/licenses/by/4.0/), which permits unrestricted use, distribution, and 
to controls (13.86\%) [7]. Up to now, this has been the only clinical research looking into the relationship between $T$. gondii infection and NAFLD. However, the data was from eastern China and the sample size was relatively small and poorly representative. It is important to explore this relationship further in a different population with a larger sample size. Determining this association between $T$. gondii and NAFLD may contribute towards the further understanding and control of both diseases. This study analyzes the relationship between $T$. gondii infection and incidence of NAFLD on a population-based dataset from the United States.

\section{Methods}

\section{Study population}

This cross-sectional study was based on the data from The Third National Health and Nutrition Examination Surveys (NHANES III) [11]. The NHANES III is a periodic survey conducted by the National Center between 1988 and 1994, and is the only survey that contains data with both liver ultrasonography examination and anti- $T$. gondii antibodies tests.

Participants who had undergone both ultrasonography examination and serum anti-T. gondii antibody tests were selected as cases. Those patients with a history of alcohol consumption and patients with chronic viral hepatitis B or $C$ were excluded.

This study was approved by the Research Ethics Review Board. The informed consents were obtained from all subjects as described in the original research design [12]. All data and further information on NHANES are available on the website [11].

\section{Demographic variables}

Age, sex, and body mass index (BMI) were collected. BMI was calculated as weight (in kilograms) divided by the square of the height (in meters) and classified as underweight $\left(<18.5 \mathrm{~kg} / \mathrm{m}^{2}\right)$, normal weight $\left(18.5-24.9 \mathrm{~kg} / \mathrm{m}^{2}\right)$, overweight $\left(25.0-29.9 \mathrm{~kg} / \mathrm{m}^{2}\right)$, obese class I $\left(30.0-34.9 \mathrm{~kg} / \mathrm{m}^{2}\right)$, and obese class II $\left(\geq 35.0 \mathrm{~kg} / \mathrm{m}^{2}\right)$ [13].

\section{Laboratory measurements}

Serum cholesterol, triglyceride, urea nitrogen, serum creatinine, fasting blood-glucose (FBG) and uric acid were obtained from original datasets. To be more specific, the blood from each participant was processed, stored and shipped to the Centers for Disease Control and Prevention for further biochemical analysis. All the sera were tested by the same method. The anti-T. gondii antibodies were tests using indirect enzyme immunoassay (EIA). The diagnostic threshold was $7 \mathrm{IU} / \mathrm{mL}$ as described in the original document. The detailed methods for the testing of anti- $T$. gondii antibodies can be retrieved from the website [14].

\section{Identification of NAFLD}

The level of hepatic steatosis was assessed by reviewing ultrasound video images of hepatic/gallbladder based on the following criteria: 1) the brightness levels of the liver parenchyma, 2) the existence of liver-to-kidney contrast, 3) the existence of deep beam attenuation, 4) the definition of the gallbladder walls, and 5) the existence of echogenic walls in the small intrahepatic vessels [15]. Participants were divided into normal to mild NAFLD, as well as moderate or severe NAFLD according to the results of liver ultrasonography [16].

\section{Statistical analysis}

The categorical variables were expressed as a percentage while continuous data were expressed as mean \pm standard deviation. The Chi-square test was used to compare the categorical variable whilst the t-test was used to compare the continuous variables. The potential risk factors of NAFLD were explored using Logistic regression with the enter method. A $P$ value $<0.05$ in univariate analysis were selected for multivariate analysis. All tests were two-tails and results with a $P$ value $<0.05$ was considered statistically significant. All analysis was conducted by R 3.4.4 [17].

\section{Results}

\section{Characteristics of participants}

A total of 12,378 participants who received both ultrasound examination and serum tests were eligible. After excluding 2613 individuals with a history of alcohol consumption and 300 cases of viral hepatitis, a total of 9465 persons were included in the final analysis. The mean age of the patients was $44.33 \pm 16.21(20-74)$ years, with 4441 (46.9\%) males and 5024 (53.1\%) females. The mean BMI of the patients enrolled was $27.60 \pm 5.96 \mathrm{~kg} / \mathrm{m}^{2}$.

\section{Comparison of $T$. gondii seropositive group and seronegative group}

There were 2520 (26.62\%) cases who tested positive for $T$. gondii. The participants were divided into a seronegative group and a seropositive group according to the serological test for anti- $T$. gondii antibody IgG. Males had a higher infection rate than females $(28.19 \%$ vs. $25.34 \%, P<0.001)$ (Fig. 1b). Seropositive participants were older in age than seronegative ones (49.41 years vs. 42.48 years, $P<0.001$ ). There was an increasing trend of seroprevalence with age ( $\mathrm{P}$ for trend $<0.001$ ). The metabolic biomarkers, including cholesterol, triglyceride, FBG, uric acid and glycated hemoglobin (HBA1c) were significantly higher in the seropositive group than the seronegative group $(P<0.05)$. However, most biomarkers for liver cell injury, for example, ALT, AST, and TBIL were comparable between the two groups. Fig. 1a and c show that prevalence of seropositivity for T. gondii increased with age. The data showed a higher incidence of NAFLD in the seropositive group than in the 

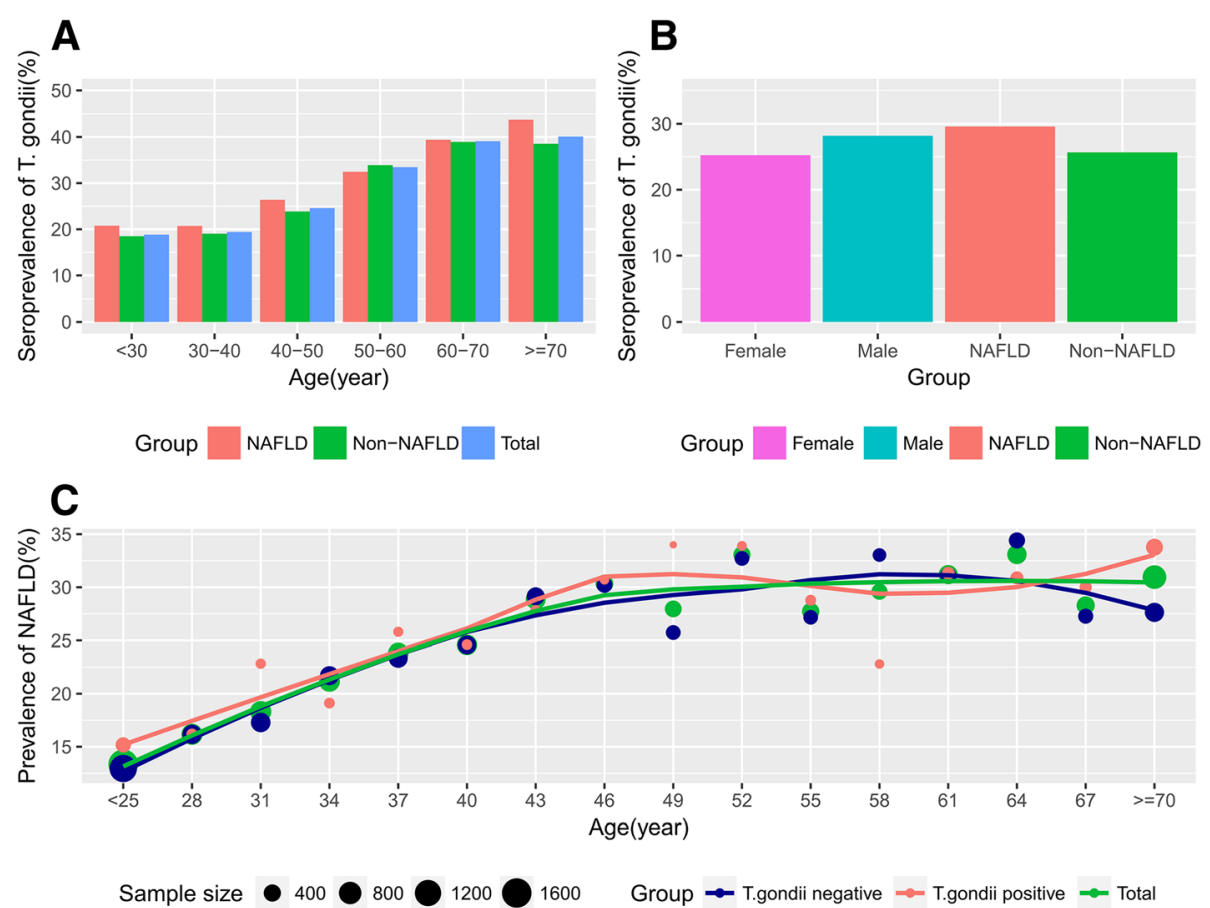

Fig. 1 Seroprevalence of T. gondii and prevalence of NAFLD. a Seroprevalence of T. gondii increases with age. In general, the NAFLD group had a slightly higher positive rate of T. gondii antibodies than the non-NAFLD group. $\mathbf{b}$ Seroprevalence of T. gondii was higher in the male and NAFLD groups. c Prevalence of NAFLD was measured according to age. The scatter plot and its loess fitted line showed a positive association between the prevalence of NAFLD and age. However, the lines representing the T. gondii positive group and the T. gondii negative group entangle, indicating that there is no significant difference in the prevalence of NAFLD between the two groups after adjusting for age

seronegative group $(27.10 \%$ vs $23.40 \%, p<0.001)$. Details are shown in Table 1.

The relationship between NAFLD and $T$. gondii antibody As shown in Table 1, the prevalence of NAFLD and older age are higher in the seropositive group. However, there was not a statistically significant difference in $T$. gondii antibody seropositivity between NAFLD and non-NAFLD groups in different age groups $(P>0.05)$ (Table 2$)$.

\section{Multivariate analysis for NAFLD}

In order to measure the relationship between $T$. gondii infection and NAFLD, multivariate logistics regression was conducted. Gender, age, BMI, T. gondii infection, serum lipid, and FBG were included in the model as independent variables. The model revealed that $T$. gondii infection was not an independent risk factor for NAFLD $(\mathrm{OR}=1.01$, the $P$ value was 0.85$)$. Higher age, FBG, and uric acid were positively associated with the probability of having NAFLD, while the effect of BMI and triglyceride experienced a first fall and then a rise in the positive association. Female $(\mathrm{OR}=0.82, P$ value $<0.001)$ turned to be a protective factor for the onset of NAFLD. See details in Table 3.

\section{Discussion}

The infection rate of $T$. gondii still remains greater than $10 \%$ in the United States even though there has been a slightly decline in seroprevalence of $T$. gondii from $13.2 \%$ in 2009-2010 [18] to $11.14 \%$ in 2011-2014 [19]. On the other hand, the prevalence of NAFLD is as high as 30\% in the same population [20,21]. Our study showed a higher incidence of NAFLD in the T. gondii seropositive group than in the seronegative group $(27.10 \%$ vs $23.40 \%, p<$ $0.001)$. However, multivariate regression indicated that infection might not be an independent risk for NAFLD.

Evidence from basic and clinical studies indicated the T. gondii infection might be responsible for liver steatosis [7, 8]. A significant change in pathology has been found in mice infected with $T$. gondii, including inflammatory cell infiltration, hepatocyte necrosis and hepatosteatosis [10]. Further transcriptomic analysis of $T$. gondii infected mice showed downregulation of peroxisome proliferator-activated receptors signaling pathway in the liver [10], which has long been proven to play key roles in regulating host bile biosynthesis, fatty acid metabolism, lipid metabolism and energy metabolism [22]. Besides the direct influence in the liver, $T$. gondii infection may play a role in diabetes mellitus, which is a well-known risk factor of NAFLD [23-25]. T. gondii infected mice had a significant reduction of pancreatic islet cells, as well as an apparent decrease in insulin expression 
Table 1 Baseline demographics of the study population

\begin{tabular}{|c|c|c|c|c|}
\hline \multirow[t]{3}{*}{ Variables } & \multicolumn{2}{|c|}{ Anti- T. gondii antibody lgG } & \multirow[t]{3}{*}{$t / x^{2}$} & \multirow{3}{*}{$\begin{array}{l}p \\
\text { value }\end{array}$} \\
\hline & \multirow{2}{*}{$\begin{array}{l}\text { Negative } \\
(n=6945)\end{array}$} & \multirow{2}{*}{$\begin{array}{l}\text { Positive } \\
(n=2520)\end{array}$} & & \\
\hline & & & & \\
\hline Age (years) ${ }^{a}$ & $42.48 \pm 15.76$ & $49.41 \pm 16.37$ & -18.71 & $<0.001$ \\
\hline \multicolumn{5}{|l|}{ Age groups (\%) } \\
\hline$<30$ years & 1982 (28.54\%) & $460(18.25 \%)$ & 345.87 & $<0.001$ \\
\hline $30-40$ years & 1623(23.37\%) & $391(15.52 \%)$ & & \\
\hline 40-50 years & 1165(16.77\%) & $380(15.08 \%)$ & & \\
\hline $50-60$ years & 839(12.08\%) & $422(16.75 \%)$ & & \\
\hline $60-70$ years & $985(14.18 \%)$ & $632(25.08 \%)$ & & \\
\hline$\geq 70$ years & $351(5.05 \%)$ & $235(9.33 \%)$ & & \\
\hline \multicolumn{5}{|l|}{ Gender (\%) } \\
\hline Male & $3189(45.92 \%)$ & 1252(49.68\%) & 10.52 & 0.001 \\
\hline Female & $3756(54.08 \%)$ & $1268(50.32 \%)$ & & \\
\hline Ethnicity (\%) & & & 43.56 & $<0.001$ \\
\hline Non-Hispanic white & 2375(34.20\%) & $907(35.99 \%)$ & & \\
\hline Non-Hispanic black & 2042(29.40\%) & $696(27.62 \%)$ & & \\
\hline Mexican American & 2293(33.02\%) & $761(30.20 \%)$ & & \\
\hline Others & $235(3.38 \%)$ & $156(6.19 \%)$ & & \\
\hline $\mathrm{BMI}\left(\mathrm{kg} / \mathrm{m}^{2}\right)$ & $27.47 \pm 6.00$ & $27.96 \pm 5.85$ & -3.48 & $<0.001$ \\
\hline \multicolumn{5}{|l|}{ NAFLD (\%) } \\
\hline Yes & $1625(23.40 \%)$ & $683(72.90 \%)$ & 13.77 & $<0.001$ \\
\hline No & $5320(76.60 \%)$ & $1837(27.10 \%)$ & & \\
\hline Cholesterol $(\mathrm{mmol} / \mathrm{L})^{\mathrm{a}}$ & $5.38 \pm 1.17$ & $5.62 \pm 1.20$ & -8.53 & $<0.001$ \\
\hline Triglyceride $(\mathrm{mmol} / \mathrm{L})^{a}$ & $1.64 \pm 1.33$ & $1.81 \pm 1.42$ & -5.27 & $<0.001$ \\
\hline $\mathrm{FBG}(\mathrm{mmol} / \mathrm{L})^{\mathrm{a}}$ & $5.47 \pm 2.09$ & $5.80 \pm 2.52$ & -6.54 & $<0.001$ \\
\hline Uric acid $(\mathrm{mmol} / \mathrm{L})^{\mathrm{a}}$ & $311.40 \pm 92.37$ & $322.18 \pm 95.15$ & -4.98 & $<0.001$ \\
\hline $\operatorname{HbA1c}(\%)^{a}$ & $5.54 \pm 1.15$ & $5.73 \pm 1.27$ & -6.95 & $<0.001$ \\
\hline TBIL $(\mathrm{mmol} / \mathrm{L})^{\mathrm{a}}$ & $9.92 \pm 5.55$ & $9.67 \pm 5.00$ & 2.01 & 0.044 \\
\hline $\operatorname{ALT}(U / L)^{a}$ & $18.32 \pm 15.36$ & $17.96 \pm 17.44$ & 0.96 & 0.34 \\
\hline $\mathrm{AST}(\mathrm{U} / \mathrm{L})^{\mathrm{a}}$ & $21.73 \pm 14.09$ & $21.97 \pm 14.18$ & -0.72 & 0.47 \\
\hline GGT $(U / L)^{a}$ & $25.72 \pm 39.28$ & $28.45 \pm 53.48$ & -2.69 & 0.007 \\
\hline $\operatorname{ALP}(U / L)^{a}$ & $86.24 \pm 27.85$ & $91.41 \pm 38.98$ & -7.08 & $<0.001$ \\
\hline Albumin $(g / L)^{a}$ & $41.52 \pm 3.87$ & $41.16 \pm 3.84$ & 4.03 & $<0.001$ \\
\hline Globulin $(\mathrm{g} / \mathrm{L})^{\mathrm{a}}$ & $33.14 \pm 4.62$ & $33.72 \pm 4.82$ & -4.69 & $<0.001$ \\
\hline Creatinine $(u m o l / L)^{a}$ & $93.27 \pm 26.13$ & $96.35 \pm 32.40$ & -4.71 & $<0.001$ \\
\hline
\end{tabular}

Abbreviations: FBG fasting blood-glucose, HBA1C Glycated hemoglobin, TBIL total bilirubin, ALT alanine transaminase, AST aspartate aminotransferase, GGT gamma-glutamyl transpeptidase, $A L P$ alkaline phosphatase ${ }^{a}$ Express as mean \pm sd or number(\%)

[23]. The results from this study also demonstrated higher BMI, cholesterol, triglyceride, uric acid and FBG levels in $T$. gondii seropositive group, also suggesting an effect of T. gondii infection on metabolism. All these mechanisms might contribute to the higher NAFLD levels seen in the T. gondii antibody seropositive population.
Table 2 Prevalence of NAFLD in the different T. gondii groups after age stratification

\begin{tabular}{|c|c|c|c|c|c|}
\hline & \multicolumn{2}{|l|}{ Non-NAFLD } & \multicolumn{2}{|l|}{ NAFLD } & \multirow[b]{2}{*}{$p$ value } \\
\hline & $\begin{array}{l}\text { No. T. gondii } \\
\text { positive }\end{array}$ & $\begin{array}{l}\text { T. gondii } \\
\text { prevalence }\end{array}$ & $\begin{array}{l}\text { No. T. gondii } \\
\text { positive }\end{array}$ & $\begin{array}{l}\text { T. gondii } \\
\text { prevalence }\end{array}$ & \\
\hline \multicolumn{6}{|c|}{ Age(years) } \\
\hline$<30$ & 385 & $18.50 \%$ & 75 & $20.78 \%$ & 0.31 \\
\hline $30-40$ & 300 & $19.05 \%$ & 91 & $20.73 \%$ & 0.43 \\
\hline $40-50$ & 262 & $23.86 \%$ & 118 & $26.40 \%$ & 0.29 \\
\hline $50-60$ & 297 & $33.90 \%$ & 125 & $32.47 \%$ & 0.62 \\
\hline $60-70$ & 435 & $38.94 \%$ & 197 & $39.40 \%$ & 0.86 \\
\hline$\geq 70$ & 158 & $38.54 \%$ & 77 & $43.75 \%$ & 0.24 \\
\hline
\end{tabular}

However, in multivariate analysis, T. gondii infection was not an independent factor for NAFLD. Unmeasured confounders may explain the association between NAFLD and T. gondii. Although basic and clinical studies had shown an increased risk of NAFLD in T. gondii infected patients, the results of this study found that, rather than T. gondii infection, only age, gender, BMI, uric acid, fasting blood-glucose and serum lipid levels were independent risk factors for the presence of NAFLD. This might be because T. gondii infection alone is a confounder which is associated with other NAFLD-related variables, such as obesity, diabetes or hyperlipidemia [25-27]. The prevalence of $T$. gondii rises with age $[18,19]$. As shown in Fig. 1c, the incidence of NAFLD also increases with age, regardless of the presence of $T$. gondii antibody. It is possible that the positive correlation between NAFLD and T. gondii is indirect, and that age plays a key role in it. These questions require further exploration through basic research.

However, the direction of the causal relationship between NAFLD and T. gondii infection might be opposite. Based on this cross-sectional study, we could not rule out the possibility that NAFLD might increase the risk of $T$. gondii infection. Patients with chronic liver diseases, including NAFLD, are susceptible to various pathogens infection [8]. Those patients have a depressed immune response in both cell-mediated and humoral immunity [20]. As a result, they might have a remarkably declined ability to protect the hosts against $T$. gondii infection. Several researches have shown a higher seroprevalence of $T$. gondii in patients with chronic liver diseases than those without in China and Egypt [7, 8]. In this study, the NAFLD patients also have an increasing $T$. gondii seropositive rate $(29.6 \%$ vs. $25.7 \%, p<0.001$, data not shown). Those studies, including the present one, suggest the possibility that NAFLD is a risk factor for $T$. gondii infection cannot be excluded. However, a small-sized study from Mexico including 75 adults with liver disease (approximately 50\% were alcoholic 
Table 3 Multivariate logistics analysis for associations between risk factors and NAFLD

\begin{tabular}{|c|c|c|c|}
\hline Variables & Adjusted OR & $95 \% \mathrm{Cl}$ & $p$ value \\
\hline \multicolumn{4}{|l|}{ Gender } \\
\hline Male & 1 & - & - \\
\hline Female & 0.82 & $0.74-0.91$ & $<0.001$ \\
\hline \multicolumn{4}{|l|}{ Age(year) } \\
\hline$<30$ & 1 & - & - \\
\hline $30-50$ & 1.46 & $1.26-1.68$ & $<0.001$ \\
\hline$\geq 50$ & 1.61 & $1.39-1.88$ & $<0.001$ \\
\hline \multicolumn{4}{|l|}{$\mathrm{BMI}\left(\mathrm{kg} / \mathrm{m}^{2}\right)$} \\
\hline$<18.5$ & 1 & - & - \\
\hline $18.5-30$ & 0.89 & $0.58-1.37$ & 0.6 \\
\hline$\geq 30$ & 2.4 & $1.55-3.72$ & $<0.001$ \\
\hline \multicolumn{4}{|c|}{ Anti- T. gondii antibody lgG } \\
\hline Negative & 1 & - & - \\
\hline Positive & 1.01 & $0.90-1.13$ & 0.85 \\
\hline \multicolumn{4}{|c|}{ Cholesterol(mmol/L) } \\
\hline$<2.9$ & 1 & - & - \\
\hline $2.9-6$ & 0.63 & $0.30-1.33$ & 0.23 \\
\hline$\geq 6$ & 0.66 & $0.31-1.41$ & 0.29 \\
\hline \multicolumn{4}{|c|}{ Triglyceride(mmol/L) } \\
\hline$<0.45$ & 1 & - & - \\
\hline $0.45-1.69$ & 0.75 & $0.66-0.86$ & $<0.001$ \\
\hline$\geq 1.69$ & 1.68 & $1.46-1.92$ & $<0.001$ \\
\hline \multicolumn{4}{|l|}{$\mathrm{FBG}(\mathrm{mmol} / \mathrm{L})$} \\
\hline$<3.9$ & 1 & - & - \\
\hline $3.9-5.6$ & 1.31 & $0.78-2.20$ & 0.31 \\
\hline$\geq 5.6$ & 2.41 & $1.42-4.07$ & $<0.001$ \\
\hline \multicolumn{4}{|c|}{ Uric acid(umol/L) } \\
\hline$<149$ & 1 & - & - \\
\hline $149-416$ & 1.66 & $0.24-0.88$ & 0.12 \\
\hline$\geq 416$ & 2.19 & $0.66-0.87$ & 0.02 \\
\hline
\end{tabular}

liver diseases) and 150 controls failed to demonstrate a relationship between seroprevalence of anti- $T$. gondii antibodies and liver disease [28]. Therefore, the complex relationship between $T$. gondii and NAFLD requires further research.

The limitation of this study was that data were collected from 1988 to 1994 when the prevalence of T. gondii was different from recent years. However, as far as we know, it was the largest population-based investigation containing datasets of both NAFLD and anti- T. gondii antibodies. Nevertheless, our results clarify the relationship between T. gondii infection and NAFLD to some extent and offer interesting and useful evidence to further the understanding of both diseases.

\section{Conclusions}

In conclusion, patients with $T$. gondii infection have a higher prevalence of NAFLD. Age may have an effect on the increase of NAFLD in the T. gondii seropositive population.

\section{Abbreviations}

ALP: Alkaline phosphatase; ALT: Alanine transaminase; AST: Aspartate aminotransferase; FBG: Fasting blood-glucose; GGT: Gamma-glutamyl transpeptidase; HBA1c: Glycated hemoglobin; TBIL: Total bilirubin

\section{Acknowledgments}

We thank Yulan Lin, MD, Ph.D., senior statistician, from School of Public Health, Fujian Medical University, China, for thoroughly reviewing our study methods and statistical analysis.

\section{Funding}

The Medical Innovation Project of Fujian Province (grant no. 2016-CX-33) and the Natural Science Foundation of Fujian Province the Pilot of Fujian Science and Technology Project (grant no. 2016Y0040, no.2017 J01187) supported the analysis of the data and writing of the manuscript.

\section{Availability of data and materials}

All data generated or analyzed during this study are included in the published article.

\section{Authors' contributions \\ $\mathrm{JH}$ did the data collection and wrote the primary draft. SL did the study BW collected data and analyzed the data. SL and MW were involved in manuscript preparation and data collection. YZ contributed to supervising the project and checked the final version of the manuscript. All authors read and approved the final version of the manuscript. \\ Ethics approval and consent to participate \\ Not applicable. \\ Consent for publication \\ Not applicable. \\ Competing interests \\ The authors declare that they have no competing interests.} design and revised the article for important intellectual content. $\mathrm{HZ}, \mathrm{BV}$, and

\section{Publisher's Note}

Springer Nature remains neutral with regard to jurisdictional claims in published maps and institutional affiliations.

\section{Author details}

${ }^{1}$ Department of Liver Research Center, the First Affiliated Hospital of Fujian Medical University, Fuzhou, No. 20, Chazhong Road, Taijiang District, Fuzhou 350001, Fujian, China. ${ }^{2}$ School of Public Health, Sun Yat-sen University, Guangzhou 350002, Guangdong, China. ${ }^{3}$ Faculty of Life Sciences and Medicine, King's College London, London SE1 1UL, UK. ${ }^{4}$ Basildon and Thurrock University Hospitals NHS Foundation Trust, Nethermayne, Basildon, Essex SS16 5NL, UK

Received: 8 August 2018 Accepted: 23 November 2018

Published online: 06 December 2018

\section{References}

1. Montoya JG, Liesenfeld O. Toxoplasmosis. Lancet. 2004;363(9425):1965-76.

2. Chorlton S. Toxoplasma gondii and schizophrenia: a review of published RCTs. Parasitol Res. 2017;116(7):1793-9.

3. Ansarilari M, Farashbandi H, Mohammadi F. Association of Toxoplasma gondii infection with schizophrenia and its relationship with suicide attempts in these patients.Tropical Med Int Health. 2017;22(10):1322-27.

4. Abd El-Rehim El-Henawy A, Abdel-Razik A, Zakaria S, Elhammady D, Saudy N, Azab MS. Is toxoplasmosis a potential risk factor for liver cirrhosis? Asian Pac J Trop Med. 2015;8(10):784-91. 
5. Da Silva AS, Tonin AA, Thorstenberg ML, Leal DB, Fighera R, Flores MM, et al. Relationship between butyrylcholinesterase activity and liver injury in mice acute infected with Toxoplasma gondii. Pathol Res Pract. 2013;209(2):95-8.

6. El-Nahas HA, El-Tantawy NL, Farag RE, Alsalem AM. Toxoplasma gondii infection among chronic hepatitis C patients: a case-control study. Asian Pac J Trop Med. 2014;7(8):589-93.

7. Tian AL, Li GX, Elsheikha HM, Gardner DS, Zhang XY, Dong W, et al. Seroepidemiology of Toxoplasma gondii infection in patients with liver disease in eastern China. Epidemiol Infect. 2017;145(11):2296-302.

8. El-Sayed NM, Ramadan ME, Ramadan ME. Toxoplasma gondii infection and chronic liver diseases: evidence of an association. Trop Med Infect Dis. 2016; (1):7.

9. Chalasani N, Younossi Z, Lavine JE, Charlton M, Cusi K, Rinella M, et al. The diagnosis and management of nonalcoholic fatty liver disease: practice guidance from the American Association for the Study of Liver Diseases. Hepatology. 2018;67(1):328-57.

10. He JJ, Ma J, Elsheikha HM, Song HQ, Huang SY, Zhu XQ. Transcriptomic analysis of mouse liver reveals a potential hepato-enteric pathogenic mechanism in acute Toxoplasma gondii infection. Parasit Vectors. 2016;9(1):427.

11. National Center for Health Statistics. https://wwwn.cdc.gov/nchs/nhanes/ nhanes3/Default.aspx. Accessed 1 Dec 1996.

12. Mazidi M, Penson P, Banach M. Association between telomere length and complete blood count in US adults. Arch Med Sci. 2017;13(3):601-5.

13. Howell CR, Fontaine K, Ejima K, Ness KK, Cherrington A, Mehta T. Maximum Lifetime Body Mass Index and Mortality in Mexican American Adults: the National Health and Nutrition Examination Survey III (1988-1994) and NHANES 1999-2010. Prev Chronic Dis. 2017;14:E67.

14. Laboratory File. https://wwwn.cdc.gov/nchs/data/nhanes3/1a/lab-acc.pdf. Accessed 1 Dec 1996.

15. Hepatic/Gallbladder Ultrasound and Hepatic Steatosis, https://wwwn.cdc. gov/nchs/data/nhanes3/34a/HGUHS.htm. Accessed 1 Jan 2011.

16. Pan M, Lyu C, Zhao J, Shen B. Sixty years (1957-2017) of research on toxoplasmosis in China-an overview. Front Microbiol. 2017;8:1825.

17. The R Project for Statistical Computing. https://www.r-project.org/. Accessed 2 July 2018.

18. Jones JL, Kruszon-Moran D, Rivera HN, Price C, Wilkins PP. Toxoplasma gondii seroprevalence in the United States 2009-2010 and comparison with the past two decades. Am J Trop Med Hyg. 2014;90(6):1135-9.

19. Jones JL, Kruszon-Moran D, Elder S, Rivera HN, Press C, Montoya JG, et al. Toxoplasma gondii infection in the United States, 2011-2014. Am J Trop Med Hyg. 2018;98(2):551-7.

20. Reccia I, Kumar J, Akladios C, Virdis F, Pai M, Habib N, et al. Non-alcoholic fatty liver disease: a sign of systemic disease. Metabolism. 2017;72:94-108.

21. Augustin S, Graupera I, Caballeria J. Non-alcoholic fatty liver disease: A poorly known pandemic. Med Clin (Barc). 2017;149(12):542-49.

22. Silva AKS, Peixoto CA. Role of peroxisome proliferator-activated receptors in non-alcoholic fatty liver disease inflammation. Cell Mol Life Sci. 2018;75(16): 2951-61.

23. Nassief Beshay EV, El-Refai SA, Helwa MA, Atia AF, Dawoud MM. Toxoplasma gondii as a possible causative pathogen of type-1 diabetes mellitus: evidence from case-control and experimental studies. Exp Parasitol. 2018;188:93-101.

24. Han Y, Nie L, Ye X, Zhou Z, Huang S, Zeng C, et al. The association between Toxoplasma gondii infection and hypertensive disorders in T2DM patients: a case-control study in the Han Chinese population. Parasitol Res. 2018;117(3): 689-95.

25. Majidiani H, Dalvand S, Daryani A, Galvan-Ramirez ML, Foroutan-Rad M. Is chronic toxoplasmosis a risk factor for diabetes mellitus? A systematic review and meta-analysis of case-control studies. Braz J Infect Dis. 2016;20(6):605-9.

26. Alvarado-Esquivel C, Maldonado-Soto E, Sanchez-Anguiano LF, Hernandez-Tinoco J, Ramos-Nevarez A, Cerrillo-Soto SM, et al. Lack of serological and molecular association between Toxoplasma gondii exposure and obesity: a case-control study. Int J Biomed Sci. 2017;13(2):74-8.

27. Reeves GM, Mazaheri S, Snitker S, Langenberg P, Giegling I, Hartmann AM, et al. A positive association between T. gondii seropositivity and obesity. Front Public Health. 2013;1:73.

28. Alvarado-Esquivel C, Torres-Berumen $J \mathrm{~L}$, Estrada-Martinez S, Liesenfeld O, Mercado-Suarez MF. Toxoplasma gondii infection and liver disease: a casecontrol study in a northern Mexican population. Parasit Vectors. 2011:4:75.

\section{Ready to submit your research? Choose BMC and benefit from:}

- fast, convenient online submission

- thorough peer review by experienced researchers in your field

- rapid publication on acceptance

- support for research data, including large and complex data types

- gold Open Access which fosters wider collaboration and increased citations

- maximum visibility for your research: over $100 \mathrm{M}$ website views per year

At $\mathrm{BMC}$, research is always in progress.

Learn more biomedcentral.com/submissions 